\title{
The Management of Positive Axillary Nodes in Early Stage Breast Cancer: Analysis of Practice Pattern Concordance with Landmark Trials
}

\author{
Danushka Seneviratne ${ }^{1}$, Laura Vallow ${ }^{1, ~ *, ~ J i n n y ~ G u n n ², ~ T a m m e z a ~ G i b s o n ~}{ }^{2}$, Emmanuel Gabriel $^{2}$, \\ Sanjay Bagaria ${ }^{2}$, Sarah McLaughlin ${ }^{2}$ \\ ${ }^{1}$ Department of Radiation Oncology, Mayo Clinic, Jacksonville, Florida, United States \\ ${ }^{2}$ Department of Surgery, Mayo Clinic, Jacksonville, Florida, United States
}

Email address:

Vallow.Laura@mayo.edu (L. Vallow),Seneviratne.danushka@mayo.edu (D. Seneviratne)

${ }^{*}$ Corresponding author

\section{To cite this article:}

Danushka Seneviratne, Laura Vallow, Jinny Gunn, Tammeza Gibson, Emmanuel Gabriel, Sanjay Bagaria, Sarah McLaughlin. The Management of Positive Axillary Nodes in Early Stage Breast Cancer: Analysis of Practice Pattern Concordance with Landmark Trials. Journal of Surgery. Vol. 9, No. 3, 2021, pp. 114-120. doi: 10.11648/j.js.20210903.14

Received: March 9, 2021; Accepted: May 5, 2021; Published: May 26, 2021

\begin{abstract}
Axillary nodal dissections (ALND) were historically performed in the management of early stage, node-positive breast cancer. Since the ACOSOG Z0011 landmark study demonstrated that patients with clinically negative axillae and 1-2 positive sentinel lymph nodes could be spared the morbidity of an axillary dissection, surgeons have widely adopted the omission of ALND. However, the practice patterns in the adjuvant setting remain less clear, and our current work wishes to address this gap by assessing the management of early stage breast cancer at our institution. We performed a retrospective analysis of 504 women with cT1/T2 N0 breast cancer undergoing breast conserving therapy (BCT) between 5/2011-6/2016 and collected data regarding the clinico-pathological characteristics of the tumors and the adjuvant therapies received. Overall, 97\% patients completed a SLNB, while 3\% underwent upfront ALND. None of SLNB positive patients had further axillary surgery. In the SLNB positive cohort however, adjuvant therapies consisted of $61.5 \%$ patients receiving regional nodal irradiation. Further analysis revealed that factors such as tumor type, $\mathrm{T}$ and $\mathrm{N}$ stage, hormone receptor status, tumor grade, the presence of LVSI, patient age, and patient race, did not correlate with clinician decisions to deviate from ACOSOG protocol recommendations regarding adjuvant therapy. Our work suggests that although ACOSOG Z0011 recommendations for minimizing axillary surgery in patients with limited nodal disease has largely been adopted at our institution, node directed radiation therapy continues to be relatively commonplace. Interestingly, the delivery of nodal irradiation was not associated with common clinic-pathological factors.
\end{abstract}

Keywords: Axillary Management, Early Stage Breast Cancer, ACOSOG Z0011

\section{Introduction}

Breast cancer is the most common malignancy impacting women within the Unites states with a large portion of patients with T1-T2 tumors and clinically negative axilla [1]. The initial management of early stage patients without clinical nodal disease involves performing a lumpectomy and axillary sentinel lymph node biopsy (SLNB) $[2,3]$. These patients typically undergo tangent based radiotherapy to the whole breast without irradiation of the regional axillary nodes $[4,5]$. Axillary nodal dissection is rarely utilized in this patient subset as a frontline treatment and is typically reserved for those with clinical evidence of axillary disease or those with more than 3 nodes positive on SLNB [5]. These treatment patterns were adopted largely based on the practice changing American College of Surgeons Oncology Group (ACOSOG) Z0011 study which indicated that in patients with small tumors and 1-2 positive SLNs, there was no significant difference in outcomes between upfront SLNB vs. ALND. In this non-inferiority landmark trial patients with 1-2 positive lymph nodes were randomized to undergo ALND or no further surgery. The protocol mandated that whole breast radiation (tangents targeting the breast) without dedicated nodal irradiation (via high tangents or a third radiation field) be performed following surgery. Initially reported results at 
6.3 years showed that avoiding ALND in patients with 1-2 positive sentinel lymph nodes did not significantly impact their disease free survival $(78.2 \%$ in the ALND arm vs. $80.2 \%$ in the SLNB only arm) or overall survival ( $83.6 \%$ in the ALND arm vs. $86.3 \%$ in the SLNB only arm) [6]. Long term follow up confirmed these findings with nodal recurrence occurring in less than $1 \%$ of patients in either group. The data from this study was heralded as paradigm shifting and many clinicians began limiting the performance of ALND in select low risk patients [7].

In contrast to the ACOSOG Z0011 trial, the AMAROS trial randomized patients with T1-T2 primary breast cancer and clinically negative axillae and positive sentinel lymph nodes to receive either an axillary dissection or axillary radiotherapy with primary endpoint of axillary recurrence. This trial found that both axillary dissection and axillary radiotherapy after a positive sentinel node allowed for excellent loco-regional disease control, while axillary radiotherapy led to significantly less patient morbidity with regard to healing and lymphedema development [8]. Many clinicians have now preferred to perform axillary radiotherapy in place of dissections to allow for disease control while minimizing side effects $[4,5]$.

Review of surgical data since the publication of ACOSOG Z0011 indicate that surgeons largely began avoiding axillary nodal dissections with 1-2 positive lymph nodes given the lack of a clear oncological benefit of ALND and the higher risk of lymphadenopathy [9]. There is however limited data about the national practice patterns with regard to adjuvant radiation delivery in this patient subset. It remains unclear whether clinicians lean on ACOSOG Z0011 recommendations of avoiding further axillary treatment or AMAROS recommendations of performing axillary radiotherapy, when treating this relatively low-risk patient cohort with early stage disease.

Our current work describes the patterns of care at our institution for early stage breast cancer patients with clinically node negative axillae. We attempted to determine whether adjuvant radiation and systemic therapy delivered in patients with 1-2 positive sentinel lymph nodes without an ALND coincides with the ACOSOG Z0011 protocol guidelines. We aimed to also document the extent of radiation therapy protocol violations during the period of 2011-2016, in order to gauge the level of clinician comfort with eliminating axillary radiation in SLNB positive patients. Furthermore, we attempted to determine whether certain high risk clinic-pathological factors played a role in the decision to perform radiation treatment differently from the ACOSOG Z0011 protocol.

\section{Methods}

After Institutional Review Board approval, we retrospectively reviewed the charts of women who underwent unilateral breast conserving surgery (BCS) and sentinel node biopsy (SLNB) between 5/2011-6/2016. We excluded patients who had prior axillary surgery, those who had neoadjuvant chemotherapy, had lymph node positive disease, or patients who ultimately underwent a mastectomy due to positive margins, leaving a study cohort of 504 women.

We gathered clinical, demographic, and pathological data on all patients. We also collected data regarding the adjuvant therapies received by these patients. Adjuvant radiotherapy was further classified according to whether the patient received partial breast, (PB), whole breast (WB) and with or without regional nodal irradiation (RNI). We determined whether the patients underwent adjuvant systemic therapy in the form of endocrine therapy or chemotherapy. With regard to radiotherapy, any cases in which radiotherapy was not delivered, partial breast radiation therapy was delivered, or dedicated regional nodal irradiation was performed were considered to be treatment violations from the protocol. With regard to systemic therapy, cases in which no adjuvant chemotherapy endocrine therapy was delivered were considered to be treatment violations.

Of note, Technetium sulfur colloid was used routinely for sentinel node mapping while blue dye was used at the surgeon's discretion and sentinel node biopsy was defined as the removal of all hot, blue, and palpable lymph nodes. Two surgeons performed all surgeries in the cohort.

Statistical analysis: Descriptive statistics for categorical variables were reported as frequencies and percentages while continuous variables were reported as mean (standard deviation) and median (range). Multivariate logistic regression analysis was performed to determine associations between clinic-pathological factors and treatment patterns/violations. All statistical tests were two-sided with the alpha level set at 0.05 for statistical significance 4 .

\section{Results}

504 women with cT1-T2 breast cancer and clinically negative axillae undergoing BCT between 2011-2016 were assessed with regard to the clinico-pathological characteristics of their tumors and adjuvant therapies. The baseline patient-related characteristics of this patient cohort are shown in Table 1.

Table 1. The baseline characteristics of the patient cohort with T1-T2 tumors and clinically negative axillae.

\begin{tabular}{ll}
\hline & $\mathbf{( N = 5 0 4 )}$ \\
\hline Age at diagnosis & \\
Median (Range) & $64.00(34.00,93.00)$ \\
BMI day of primary surgery & \\
Median (Range) & $27.30(17.10,64.50)$ \\
Race & \\
Missing & 11 \\
White & $444(90.1 \%)$ \\
Black & $29(5.9 \%)$ \\
Asian & $14(2.8 \%)$ \\
Other & $6(1.2 \%)$ \\
Diabetes Mellitus & \\
Yes & $63(12.5 \%)$ \\
No & $441(87.5 \%)$ \\
Smoker & \\
Yes & $196(38.9 \%)$ \\
No & $308(61.1 \%)$ \\
\hline
\end{tabular}

The breast tumor characteristics of this patient group were assessed and are described in Table 2. Of the 504 patients, invasive ductal carcinoma was observed in $316(62.7 \%)$ 
patients, DCIS in $9(1.8 \%)$, invasive lobular carcinoma in 43 $(8.5 \%)$, and a combination of invasive and in-situ disease in 126 patients $(25 \%)$. Most patients were found to have T1 tumors $(\mathrm{T} 1 \mathrm{a}=8.1 \%, \mathrm{~T} 1 \mathrm{~b}=26.8 \%, \mathrm{~T} 1 \mathrm{c}=45.6 \%)$, while 89 patients $(17.7 \%)$ had $\mathrm{T} 2$ tumors. With regard to pathologic nodal status in this clinically node negative cohort, most patients $(439 ; 87.1 \%)$ were pN0 or only had isolated tumor cells following sentinel nodal assessment. A relatively small number of patients $(59 ; 11.7 \%)$ had $\mathrm{pN} 1$ or $\mathrm{pN} 1 \mathrm{mic}$ disease, and only 6 patients $(1.2 \%)$ had pathologically pN2 disease. Assessment of the hormone receptor status revealed that 435 $(86.3 \%)$ were ER positive, 51 (79.6\%) were PR positive, and $51(10.4 \%)$ were HER2 positive. Further pathological analysis revealed that 158 patients $(31.6 \%)$ had grade 1 tumors, 206 (41.2\%) had grade 2 tumors, 127 (25.4\%) had grade 3 tumors, and $44(9.3 \%)$ patients had lymphovascular invasion.

Table 2. The breast tumor characteristics of the patient cohort with T1-T2 tumors and clinically negative axillae.

\begin{tabular}{|c|c|}
\hline & Total $=504$ \\
\hline \multicolumn{2}{|l|}{ Tumor type } \\
\hline DCIS & $9(1.8 \%)$ \\
\hline IDC & $316(62.7 \%)$ \\
\hline IDC+DCIS & $126(25.0 \%)$ \\
\hline ILC & $43(8.5 \%)$ \\
\hline Other & $10(2.0 \%)$ \\
\hline \multicolumn{2}{|l|}{ T status } \\
\hline Tis & $9(1.8 \%)$ \\
\hline T1a & $41(8.1 \%)$ \\
\hline $\mathrm{T} 1 \mathrm{~b}$ & $135(26.8 \%)$ \\
\hline $\mathrm{T} 1 \mathrm{c}$ & $230(45.6 \%)$ \\
\hline $\mathrm{T} 2$ & $89(17.7 \%)$ \\
\hline \multicolumn{2}{|l|}{$\mathrm{N}$ status } \\
\hline $\mathrm{pNO} / \mathrm{NOi}+$ & $439(87.1 \%)$ \\
\hline $\mathrm{pN} 1 / \mathrm{N} 1 \mathrm{mic}$ & $59(11.7 \%)$ \\
\hline $\mathrm{pN} 2$ & $6(1.2 \%)$ \\
\hline \multicolumn{2}{|c|}{ Estrogen receptors } \\
\hline Negative & $69(13.7 \%)$ \\
\hline Positive & $435(86.3 \%)$ \\
\hline \multicolumn{2}{|c|}{ Progesterone receptors } \\
\hline Negative & $103(20.4 \%)$ \\
\hline Positive & $401(79.6 \%)$ \\
\hline \multicolumn{2}{|l|}{ HER2 } \\
\hline Unknown & 12 \\
\hline Negative & $441(89.6 \%)$ \\
\hline Positive & $51(10.4 \%)$ \\
\hline \multicolumn{2}{|l|}{ Tumor grade } \\
\hline Unknown & 4 \\
\hline 1 & $158(31.6 \%)$ \\
\hline 2 & $206(41.2 \%)$ \\
\hline 3 & $127(25.4 \%)$ \\
\hline Low & $2(0.4 \%)$ \\
\hline Int & $1(0.2 \%)$ \\
\hline High & $6(1.2 \%)$ \\
\hline \multicolumn{2}{|c|}{ Lymphovascular invasion } \\
\hline Unknown & 29 \\
\hline No & $431(90.7 \%)$ \\
\hline Yes & $44(9.3 \%)$ \\
\hline
\end{tabular}

The data regarding the surgical axillary management of this patient cohort is shown in Table 3. At the time of lumpectomy, 487 patients $(66.6 \%)$ underwent a sentinel lymph node biopsy. Upfront axillary lymph node dissection was performed only in 17 patients $(3.37 \%)$. Of those who underwent a SLN assessment first, $52 / 487$ patients $(10.67 \%)$ had $1-2$ positive nodes and had no further axillary surgery. Of those who had an upfront axillary dissection, $15 / 17$ patients $(88.23 \%)$ had node positive disease.

Table 3. Number of patients with metastatic disease within the lymph nodes following undergoing either SLNB only or upfront ALND.

\begin{tabular}{lllll}
\hline & SLNB (N=487) & ALND (N=17) & Total $(\mathbf{N}=\mathbf{5 0 4})$ & p value \\
\hline LN & & & & $<0.001$ \\
Negative & $435(99.5 \%)$ & $2(0.05 \%)$ & 437 & \\
Positive & $52(77.6 \%)$ & $15(22.4 \%)$ & 67 & \\
\hline
\end{tabular}

Table 4. The adjuvant treatment delivered for patients with T1-T2 tumors and clinically negative axillae after SLNB or ALND.

\begin{tabular}{|c|c|}
\hline & Total $=487$ \\
\hline \multicolumn{2}{|c|}{ Breast XRT Treatment Location } \\
\hline Nmiss & 14 \\
\hline None & $39(8.0 \%)$ \\
\hline Mayo & $349(71.2 \%)$ \\
\hline OSF & $102(20.8 \%)$ \\
\hline \multicolumn{2}{|c|}{ Breast XRT Treatment Type } \\
\hline Nmiss & 58 \\
\hline PB & $38(8.5 \%)$ \\
\hline WB & $362(81.2 \%)$ \\
\hline $\mathrm{WB}+\mathrm{RNI}$ & $46(10.3 \%)$ \\
\hline Chest Wall & $0(0.0 \%)$ \\
\hline Chest Wall+RNI & $0(0.0 \%)$ \\
\hline \multicolumn{2}{|c|}{ Adjuvant Chemotherapy } \\
\hline Nmiss & 12 \\
\hline None & $321(65.2 \%)$ \\
\hline $\mathrm{ACT}$ & $27(5.5 \%)$ \\
\hline ACTH & $3(0.6 \%)$ \\
\hline $\mathrm{TC}$ & $94(19.1 \%)$ \\
\hline $\mathrm{TCH}$ & $18(3.7 \%)$ \\
\hline TCHP & $2(0.4 \%)$ \\
\hline $\mathrm{TH}$ & $14(2.8 \%)$ \\
\hline Other & $13(2.6 \%)$ \\
\hline \multicolumn{2}{|c|}{ Endocrine Therapy } \\
\hline Nmiss & 11 \\
\hline None & $109(22.1 \%)$ \\
\hline Tamoxifen & $25(5.1 \%)$ \\
\hline AI & $294(60.3 \%)$ \\
\hline Both & $27(5.5 \%)$ \\
\hline Less than 1 year & $38(7.8 \%)$ \\
\hline \multicolumn{2}{|l|}{ Therapy } \\
\hline Chemo Only & $56(11.1 \%)$ \\
\hline Endocrine Only & $269(53.4 \%)$ \\
\hline Both & $115(23.6 \%)$ \\
\hline Neither & $64(12.7 \%)$ \\
\hline
\end{tabular}

Adjuvant therapy received by those who underwent sentinel nodal biopsy were assessed in detail and documented in Table 4. Most patients within this cohort received adjuvant treatment at Mayo Clinic $(349 ; 71.2 \%)$, while a smaller portion (102; $20.8 \%$ ) underwent adjuvant treatment elsewhere. No adjuvant treatment was received by 39 patients $(8.0 \%)$ and data regarding adjuvant therapies were unavailable for 14 patients. Whole breast only radiation therapy was received by 362 patients $(81.2 \%)$, whole breast with regional nodal irradiation was received by 46 patients $(10.3 \%)$, and partial breast radiation was received by 38 patients $(8.5 \%)$. Analysis of systemic therapy revealed that 56 patients $(11.1 \%)$ received chemotherapy only, 269 patients $(53.4 \%)$ received endocrine 
therapy only, and 115 patients (23.6\%) underwent both chemotherapy and endocrine therapy, while 64 patients $(13.1 \%)$ received no systemic therapy at all.

Table 5. Depicts adjuvant treatments received by those patients with 1-2 lymph nodes positive for disease following SLNB and identifies treatment violations from the ACOSOG Z 0011 protocol.

\begin{tabular}{ll}
\hline Treatment Type & Number of patients \\
\hline Breast radiation & \\
Missing & $8 / 52$ \\
PB & $1 / 52$ \\
WB & $11 / 52$ \\
WB+RNI & $32 / 52$ \\
Systemic Therapy & \\
Chemo Only & $8 / 52$ \\
Endocrine Only & $13 / 52$ \\
Both & $24 / 52$ \\
Neither & $7 / 52$ \\
Radiation treatment violations & $33 / 52$ \\
Systemic therapy treatment violations & $7 / 52$ \\
Total treatment violations & $40 / 52$ \\
\hline
\end{tabular}

The management of the subgroup of patients who fit the criteria for ACOSOG Z0011 (T1-T2 tumors, clinically node negative axilla, with 1-2 positive lymph nodes at sentinel node biopsy) was further analyzed with regard to the type of axillary surgery performed and the adjuvant therapies received. These findings are shown in Table 5. Undergoing a complete axillary dissection in the presence of 1-2 positive nodes, undergoing regional nodal irradiation, partial breast radiation, and receiving no systemic therapy were considered to be treatment violations per the ACOSOG Z0011 protocol. In this patient subset consisting of 52 patients, 32 (61.4\%) patients underwent whole breast with regional nodal irradiation, 1 $(1.9 \%)$ patient underwent partial breast radiation therapy, while $8(15.3 \%)$ patients had no radiation treatment information available, suggesting that some patients may not have received any adjuvant radiation therapy. Combining these three treatment cohorts (with potential treatment violations) leads to count of $41(78.8 \%)$ patients who may have received alternative radiation treatments from that specified in the ACOSOG Z0011 protocol. Of the patients who underwent whole breast radiation with nodal irradiation, we examined whether the presence of extranodal extension played a role in the clinician decision regarding the delivery of radiation therapy. Interestingly, only 3 patients in this cohort had pathological evidence of extranodal extension. With regard to systemic therapy, 7 (13.4\%) patients received no further adjuvant systemic therapy following lumpectomy (in the form of endocrine therapy or chemotherapy), in violation of the ACOSOG Z0011 protocol recommendations. Multivariate logistic regression analysis was then performed to determine whether certain high risk tumor pathology characteristics associated with the delivery of alternate radiation therapies that differed from the protocol recommendations. Interestingly, tumor specific factors such as tumor type, $\mathrm{T}$ stage, $\mathrm{N}$ stage, hormone receptor status, tumor grade, and LVSI was not significantly associated with the presence of radiation treatment violations. Further analysis indicated that patient specific factors such as patient age and race also was not significantly associated with the delivery with regional nodal irradiation. This data is depicted in Table 6.

Table 6. Depicts adjuvant treatments received by those patients with 1-2 lymph nodes positive for disease following SLNB and displays treatment violations from the ACOSOG Z 0011 protocol.

\begin{tabular}{|c|c|c|c|c|}
\hline & $\begin{array}{l}\text { Not a Violation } \\
(\mathrm{N}=11)\end{array}$ & $\begin{array}{l}\text { Violation } \\
(\mathrm{N}=41)\end{array}$ & $\begin{array}{l}\text { Total } \\
(\mathrm{N}=52)\end{array}$ & $\begin{array}{l}\mathbf{p} \\
\text { value }\end{array}$ \\
\hline Tumor type & & & & 0.087 \\
\hline DCIS & $0(0.0 \%)$ & $0(0.0 \%)$ & $0(0.0 \%)$ & \\
\hline IDC & $10(90.9 \%)$ & $27(65.9 \%)$ & $37(71.2 \%)$ & \\
\hline IDC+DCIS & $0(0.0 \%)$ & $12(29.3 \%)$ & $12(23.1 \%)$ & \\
\hline ILC & $1(9.1 \%)$ & $2(4.9 \%)$ & $3(5.8 \%)$ & \\
\hline Other & $0(0.0 \%)$ & $0(0.0 \%)$ & $0(0.0 \%)$ & \\
\hline $\mathrm{T}$ status & & & & 0.265 \\
\hline Tis & $0(0.0 \%)$ & $0(0.0 \%)$ & $0(0.0 \%)$ & \\
\hline T1a & $0(0.0 \%)$ & $1(2.4 \%)$ & $1(1.9 \%)$ & \\
\hline $\mathrm{T} 1 \mathrm{~b}$ & $3(27.3 \%)$ & $3(7.3 \%)$ & $6(11.5 \%)$ & \\
\hline T1c & $4(36.4 \%)$ & $24(58.5 \%)$ & $28(53.8 \%)$ & \\
\hline $\mathrm{T} 2$ & $4(36.4 \%)$ & $13(31.7 \%)$ & $17(32.7 \%)$ & \\
\hline $\mathrm{N}$ status & & & & 0.518 \\
\hline N0/N0i+ & $1(9.1 \%)$ & $2(4.9 \%)$ & $3(5.8 \%)$ & \\
\hline $\mathrm{N} 1 / \mathrm{N} 1 \mathrm{mic}$ & $10(90.9 \%)$ & $39(95.1 \%)$ & $49(94.2 \%)$ & \\
\hline $\mathrm{N} 2$ & $0(0.0 \%)$ & $0(0.0 \%)$ & $0(0.0 \%)$ & \\
\hline N3 & $0(0.0 \%)$ & $0(0.0 \%)$ & $0(0.0 \%)$ & \\
\hline $\mathrm{Nx}$ & $0(0.0 \%)$ & $0(0.0 \%)$ & $0(0.0 \%)$ & \\
\hline Estrogen receptors & & & & 0.378 \\
\hline Negative & $3(27.3 \%)$ & $6(14.6 \%)$ & $9(17.3 \%)$ & \\
\hline Positive & $8(72.7 \%)$ & $35(85.4 \%)$ & $43(82.7 \%)$ & \\
\hline $\begin{array}{l}\text { Progesterone } \\
\text { receptors }\end{array}$ & & & & 0.378 \\
\hline Negative & $3(27.3 \%)$ & $6(14.6 \%)$ & $9(17.3 \%)$ & \\
\hline Positive & $8(72.7 \%)$ & $35(85.4 \%)$ & $43(82.7 \%)$ & \\
\hline HER2 & & & & 1.000 \\
\hline Nmiss & 0 & 1 & 1 & \\
\hline Negative & $10(90.9 \%)$ & $34(85.0 \%)$ & $44(86.3 \%)$ & \\
\hline Positive & $1(9.1 \%)$ & $6(15.0 \%)$ & $7(13.7 \%)$ & \\
\hline Tumor grade & & & & 0.634 \\
\hline 1 & $2(18.2 \%)$ & $13(31.7 \%)$ & $15(28.8 \%)$ & \\
\hline 2 & $6(54.5 \%)$ & $16(39.0 \%)$ & $22(42.3 \%)$ & \\
\hline 3 & $3(27.3 \%)$ & $12(29.3 \%)$ & $15(28.8 \%)$ & \\
\hline Low & $0(0.0 \%)$ & $0(0.0 \%)$ & $0(0.0 \%)$ & \\
\hline Int & $0(0.0 \%)$ & $0(0.0 \%)$ & $0(0.0 \%)$ & \\
\hline High & $0(0.0 \%)$ & $0(0.0 \%)$ & $0(0.0 \%)$ & \\
\hline $\begin{array}{l}\text { Lymphovascular } \\
\text { invasion }\end{array}$ & & & & 0.706 \\
\hline Nmiss & 1 & 5 & 6 & \\
\hline No & $6(60.0 \%)$ & $25(69.4 \%)$ & $31(67.4 \%)$ & \\
\hline Yes & $4(40.0 \%)$ & $11(30.6 \%)$ & $15(32.6 \%)$ & \\
\hline \multicolumn{5}{|l|}{ Age } \\
\hline$<65$ & $7(72.7 \%)$ & $28(68.2 \%)$ & $34(65.3 \%)$ & 0.753 \\
\hline$>65$ & $4(36.3 \%)$ & $13(31.7)$ & $17(32.6 \%)$ & \\
\hline
\end{tabular}

\section{Discussion}

The typical management of early stage breast cancer patients with clinically negative axillae involves performing a lumpectomy with a SLNB performed at the time of surgery. The historical basis for this management strategy was shaped by several studies that indicated the oncological equivalence of SLNB to ALND. NSABP-B-32 was a clinical trial designed to determine whether SLNB led to the same survival and regional control as a complete axillary dissection. Patients were randomized to SLN resection followed by ALND, or SLN resection alone with axillary nodal dissection reserved 
only for those with positive nodes on sentinel biopsy. The authors found that overall survival, disease free survival, and regional control were equivalent between the two groups and therefore it was concluded that when SLN biopsy is negative, not performing further axillary nodal dissection is appropriate [10]. Furthermore, multiple retrospective studies and a randomized clinical trial by Purushotham et al. have shown that patients undergoing SLN biopsies experience significantly lower physical and psychological morbidly in comparison to those undergoing upfront axillary lymph node dissections [11]. Based on these studies, it was concluded that in patients with a clinically negative axillae in whom axillary staging is likely to provide clinically relevant information, a SLN biopsy should be offered first. These studies eventually paved the path for the development of the landmark ACOSOG Z0011 trial.

Given that a growing body of data demonstrated that even those patients with positive sentinel lymph node biopsies fared well in terms of loco-regional recurrence and survival, the ACOSOG Z 0011 trial was designed to assess the outcomes of patients with $<2$ positive sentinel nodes who do not undergo axillary nodal dissections or further axillary radiation therapy. The findings of the ACOSOG Z11 trial demonstrated that a complete axillary dissection can be omitted in patients with 1-2 positive lymph nodes without compromising oncological outcomes. Clinical practices across the nation have widely adopted these findings to limit toxicity $[9,12]$. Despite the interesting and widely accepted conclusions of the ACOSOG Z0011 trial, critics of the trial note that caution must be exercised when interpreting the radiation therapy data of the trial. Initial analysis of the trial revealed that among the 605 patients with completed case report form, $89 \%$ of patients underwent adjuvant whole breast radiation therapy [7]. Independent assessment revealed that a significant portion of patients received some nodal radiation therapy. Jagsi et al. performed a secondary analysis of the radiation therapy on 228 patients enrolled in the ACOSOG Z0011 trial. In this group, $18.9 \%$ of patients received protocol-prohibited nodal radiation via a third radiation field. Further analysis of 142 patients with sufficient records to assess radiation tangent field heights revealed that high tangents ((cranial tangent border $\leq 2 \mathrm{~cm}$ from humeral head) covering the axillary nodal levels I/II were used in $50 \%$ of patients who underwent ALND and 52.6\% who underwent SLND. This independent analysis regarding protocol adherence of ACOSOG Z0011 suggests that a significant portion of clinicians likely felt uncomfortable eliminating ALND and nodal irradiation in the presence of positive sentinel lymph nodes and thus performed trial prohibited nodal irradiation in an attempt to limit loco-regional recurrence [13].

Our current study aimed to characterize the treatment practices at our institution with regard to surgical and adjuvant management of patients who fit the eligibility criteria for the ACOSOG Z11 trial. Our data identified that although surgical management with SLNB without further axillary surgery in patients with 1-2 positive nodes was well adopted within our practice, adjuvant radiation and systemic therapy delivery was more variable. It appeared that most patients received regional nodal irradiation in addition to whole breast radiation therapy or no adjuvant radiation therapy at all. Interestingly however, our work did not identify an association between common high risk clinicopathological factors and the presence of radiation treatment violations.

As mentioned above, the findings of this work regarding the practice patterns at our institution appear to indicate that our surgical practice is quite stringent with regard to ACOSOG Z11 recommendations, with $0 \%$ of patients with $1-2$ positive nodes on SLNB undergoing further axillary surgery. Interestingly, large database studies indicate that surgical practices nationally appear to be more lax with regard to the enactment of further axillary surgery. For instance, a large National Cancer Database (NCDB) study of the axillary management in 83,555 patients fitting the ACOSOG Z11 eligibility criteria found that 9474 patients comprising $31.2 \%$ of the assessed cohort with 1-2 positive nodes on SLNB, had further axillary surgery [14]. In contrast, our radiation therapy practice patterns appeared to favor nodal irradiation in patients with early stage disease and no clinically apparent nodes, in accordance with the AMAROS trial which recommended performing axillary directed radiation therapy in place of axillary dissections to limit patient morbidity, while allowing for similar rates of disease control. The above mentioned NCDB database study also found that nationally, clinicians appear to favor following the ACOSOG Z11 recommendations in those patients who underwent SLNB alone without further axillary dissection, with $63.4 \%$ receiving whole breast radiation only [14]. In comparison, $36.6 \%$ underwent whole breast and nodal irradiation in the NCDB cohort, in comparison to $61.4 \%$ at our institution. The authors found that facility type and location, race, insurance status, median income and the number positive nodes associated with performing nodal irradiation in the NCDB study, while our work did not identify a relationship between the delivery of nodal irradiation and tumor pathological factors.

Although our work did not clearly identify reasoning behind why clinicians largely chose to follow AMAROS recommendations over ACOSOG Z 0011, it must be noted that the burden of nodal disease among the ACOSOG Z 0011 trial was relatively low, with $60 \%$ of patients having 1 positive node on SLN biopsy. Furthermore, in those patients who underwent ALND, 27.3\% were found to have additional positive axillary nodal disease [9]. We did consider the possibility that the presence of extranodal extension may have influenced clinician decisions to deliver radiation to the breast and the nodes. Interestingly however, given that only a small number of patients (3/32) who underwent radiation to breast and nodes had extranodal extension based on pathology review, this did not appear to be the case. These findings may have led our clinicians to be concerned that additional positive nodes would have been discovered if those patients with 1-2 positive nodes at SLNB had undergone a complete dissection, and therefore omitting localized radiation to the axilla in these undissected patients could allow for the persistence of higher 
risk disease. Moreover, there may have been clinician concern that the non-aggressive treatment approach of ACOSOG Z 0011 directly collides with certain more modern era data regarding axillary management. For instance, it must be noted that in the MA. 20 study, patients with node positive or high risk node negative breast cancer who treated with breast conserving surgery and adjuvant systematic therapy were randomized to either whole breast radiation with directed regional nodal irradiation or whole breast radiation alone. Although the addition of regional node irradiation did not improve overall survival in these patient subsets, it reduced the rate of breast cancer recurrence, improved the rate of distant metastasis free survival, and showed a trend toward improved overall survival.

INSEMA trial is another recent surgical trial that sheds light on the management of the axilla in early stage breast cancer patients. This trial randomized 5542 patients with breast conserving surgery between SLNB vs. no axillary assessment, followed by a secondary randomization in those with a positive SLNB to undergo an ALND or no further surgery. Detailed radiation records were reviewed for the first 3 patients treated at each participating radiation facility for a total of 235 sentinel lymph node positive patients. It was found that $50 \%$ of this patient cohort received $>85 \%$ of the prescribed breast dose to the axillary level I and $25 \%$ received $>75 \%$ of the prescription doe to axillary level II. This data indicated that majority of lymph node positive patients underwent some axillary lymph node radiation [15]. INSEMA trial data suggests that despite the years that have passed since the publication of the ACOSOG Z 0011 data, there remains some clinician discomfort in avoiding axillary radiation altogether in patients with 1-2 positive sentinel lymph nodes. This work is particularly interesting in the context of our current study, as our work also found that radiation oncologists prefer to deliver some regional nodal irradiation in patients with node positive, early stage breast cancer.

We found it interesting that the delivery of node directed radiation therapy was not associated with common high risk factors such as higher $\mathrm{T}$ stage, $\mathrm{N}$ stage, negative hormone receptors status, higher tumor grade or the presence of lymphovascular invasion. Additionally, common patient specific factors such as race and patient age also did not significantly associate with the delivery with regional nodal irradiation, suggesting that clinician preference towards AMAROS guidelines and/or additional patient characteristics likely played a role in this decision.

We admit that although our work sheds light into radiation practice patterns in the treatment of early stage breast cancer, the limitations of this study include its retrospective nature, the lack of data regarding whether patient/clinician preferences played a role in decisions to deliver adjuvant therapies, and the lack of information regarding Oncotype Scores, which may have influenced systemic therapy decisions. We acknowledge that further research in the form of clinician surveys and in depth analysis of additional patient factors, are necessary to better understand the presented data.
Such studies to gain better insight of the presented data and could be embarked upon in a future project.

\section{Conclusion}

Our work indicates that while there is a trend towards minimizing axillary surgery in patients with limited nodal disease on SLNB in accordance with the ACOSOG Z0011 recommendations, our radiation therapy practices appears to lean more towards the AMAROS trial recommendations of performing node directed radiation therapy in this patient subgroup.

\section{References}

[1] Bray, F., et al., Global cancer statistics 2018: GLOBOCAN estimates of incidence and mortality worldwide for 36 cancers in 185 countries. CA Cancer J Clin, 2018. 68 (6): p. 394-424.

[2] Ayala de la Pena, F., et al., SEOM clinical guidelines in early stage breast cancer (2018). Clin Transl Oncol, 2019. 21 (1): p. 18-30.

[3] Furrukh, M. and A. Qureshi, Treatment Of Breast Cancer; Review And Updates. J Ayub Med Coll Abbottabad, 2018. 30 (2): p. 264-274.

[4] Braunstein, L. Z. and J. R. Bellon, Contemporary Issues in Breast Cancer Radiotherapy. Hematol Oncol Clin North Am, 2020. 34 (1): p. 1-12.

[5] Wong, J. S., L. E. Warren, and J. R. Bellon, Management of the Regional Lymph Nodes in Early-Stage Breast Cancer. Semin Radiat Oncol, 2016. 26 (1): p. 37-44.

[6] Giuliano, A. E., et al., Axillary dissection vs no axillary dissection in women with invasive breast cancer and sentinel node metastasis: a randomized clinical trial. JAMA, 2011. 305 (6): p. 569-75.

[7] Giuliano, A. E., et al., Locoregional Recurrence After Sentinel Lymph Node Dissection With or Without Axillary Dissection in Patients With Sentinel Lymph Node Metastases: Long-term Follow-up From the American College of Surgeons Oncology Group (Alliance) ACOSOG Z0011 Randomized Trial. Ann Surg, 2016. 264 (3): p. 413-20.

[8] Donker, M., et al., Radiotherapy or surgery of the axilla after a positive sentinel node in breast cancer (EORTC 10981-22023 AMAROS): a randomised, multicentre, open-label, phase 3 non-inferiority trial. Lancet Oncol, 2014. 15 (12): p. 1303-10.

[9] Caudle, A. S., et al., American College of Surgeons Oncology Group (ACOSOG) Z0011: impact on surgeon practice patterns. Ann Surg Oncol, 2012.19 (10): p. 3144-51.

[10] Krag, D. N., et al., Sentinel-lymph-node resection compared with conventional axillary-lymph-node dissection in clinically node-negative patients with breast cancer: overall survival findings from the NSABP B-32 randomised phase 3 trial. Lancet Oncol, 2010. 11 (10): p. 927-33.

[11] Purushotham, A. D., et al., Morbidity after sentinel lymph node biopsy in primary breast cancer: Results from a randomized controlled trial. Journal of Clinical Oncology, 2005. 23 (19): p. $4312-4321$. 
[12] McCready, D., et al., Surgical management of early stage invasive breast cancer: a practice guideline. Can J Surg, 2005. 48 (3): p. 185-94.

[13] Jagsi, R., et al., Radiation field design in the ACOSOG Z0011 (Alliance) Trial. J Clin Oncol, 2014. 32 (32): p. 3600-6.

[14] Mann, J. M., et al., National Practice Patterns of Axillary
Management and Adjuvant Radiation After ACOSOG Z0011. International Journal of Radiation Oncology, 2016.

[15] Hildebrandt, G., et al., Central Review of Radiation Therapy Planning Among Patients with Breast-Conserving Surgery: Results from a Quality Assurance Process Integrated into the INSEMA Trial. Int J Radiat Oncol Biol Phys, 2020. 107 (4): p. 683-693. 